\title{
PTEN activation sensitizes breast cancer to PI3-kinase inhibitor through the $\beta$-catenin signaling pathway
}

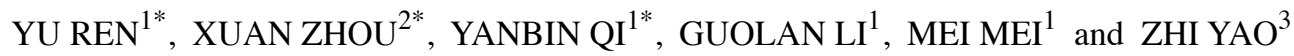 \\ ${ }^{1}$ Tianjin Research Center of Basic Medical Science, Tianjin Medical University, Tianjin 300070; ${ }^{2}$ The First Department \\ of Head and Neck Cancer, Tianjin Medical University Cancer Institute and Hospital, Tianjin 300060; ${ }^{3}$ Key Laboratory \\ of Immuno Microenviroment and Disease of the Educational Ministry, Department of Immunology, \\ Tianjin Medical University, Tianjin 300070, P.R. China
}

Received February 28, 2012; Accepted May 14, 2012

DOI: $10.3892 /$ or.2012.1856

\begin{abstract}
Combination therapy is considered a promising therapeutic modality in enhancing treatment efficacy. The phosphatidylinositol 3-kinase (PI3K)/AKT signaling pathway is almost universally dysregulated in breast cancer, with specific occurrence of PTEN mutations; thus, it has become an attractive target for cancer treatment. However, the use of single targeted therapeutics against the PI3K/AKT pathway has demonstrated only modest clinical benefits. In this study, recombinant adenovirus-mediated gene transfer of PTEN (AD-PTEN) combined with treatment with LY294002 was utilized to evaluate the effects of suppression of breast cancer cell proliferation. Herein, we show that AD-PTEN significantly enhanced the sensitization of breast cancer cells to LY294002. The 50\% inhibitory concentration $\left(\mathrm{IC}_{50}\right)$ values of LY294002 were significantly decreased to a greater extent in cells transfected with combination therapy. In addition, treatment of AD-PTEN-transfected cells with LY294002 resulted in significantly reduced cell viability and invasion ability compared to single LY294002 treatment. Using western blotting, we found that combination treatment resulted in lower levels of phosphorylated AKT ${ }^{\text {Ser473 }}$ and GSK- $3 \beta^{\text {Ser9 }}$ than single treatment with LY294002. Furthermore, we showed a significant decrease in nuclear $\beta$-catenin, Fra- 1 , Tcf- 4 and c-Myc by
\end{abstract}

Correspondence to: Dr Mei Mei, Tianjin Research Center of Basic Medical Science, Tianjin Medical University, Tianjin 300070, P.R. China

E-mail: meim11@yahoo.com.cn

Dr Zhi Yao, Key Laboratory of Immuno Microenviroment and Disease of the Educational Ministry, Department of Immunology, Tianjin Medical University, Tianjin 300070, P.R. China

E-mail: yaozhi@tijmu.edu.cn

${ }^{*}$ Contributed equally

Key words: PTEN, LY294002, $\beta$-catenin pathway, phosphatidylinositol 3-kinase/AKT pathway, breast cancer combination treatment. Our results indicate that AD-PTEN sensitization of breast cancer to LY294002 is achieved by increased GSK-3 $\beta$ activity, thus resulting in inhibition of the $\beta$-catenin signaling pathway.

\section{Introduction}

Breast cancer is a devastatingly common disease in women worldwide and there is a considerable need to improve approaches for prevention, diagnosis and treatment. Despite conventional treatments, including surgical resection, radiation therapy and chemotherapy, frequent tumor recurrence results in poor prognosis (1).

The phosphatidylinositol 3-kinase (PI3K)/AKT pathway plays an important role in the biology of human cancers. Components of this pathway are frequently deregulated in a wide range of tumors, making them attractive targets for cancer therapy $(2,3)$. Inhibitors of PI3K and AKT have undergone preclinical evaluation with encouraging results $(4,5)$.

PTEN, one of the most frequently mutated tumor suppressor genes in human cancer, shows a very high frequency of mutations in tumor cells (6). Recent studies have shown that the frequencies of breast cancer cases associated with a loss of PTEN expression are $30 \%$ in primary tumors and $25 \%$ in metastatic tumors (7). Loss of PTEN function leads to increased concentrations of $\mathrm{PIP}_{3}$, resulting in constitutive activation of downstream components of the PI3K pathway, including AKT and mTOR (8).

LY294002, a classic PI3K inhibitor, is widely used to study the PI3K/AKT signaling pathway, and has strong antitumor activity (9-11). However, most clinical trials of low-molecularweight kinase inhibitors as monotherapies have failed to demonstrate survival benefits in cancer patients. In response, combination therapy is considered a promising therapeutic model in overcoming therapeutic resistance and enhancing treatment efficacy.

In this study, adenovirus-mediated gene transfer of PTEN (AD-PTEN) was combined with LY294002 treatment and was utilized to evaluate its effect on the cell proliferation, cell cycle, invasion and migration in MCF-7 breast cancer cells. The addition of AD-PTEN reduced the $50 \%$ inhibitory concentration $\left(\mathrm{IC}_{50}\right)$ value and enhanced the antitumor effect of 
LY294002. In addition, combination therapy resulted in lower levels of phosphorylated AKT ${ }^{\text {Ser473 }}$ and GSK-3 $\beta^{\text {Ser9 }}$ than single treatment with LY294002. Several members of the Wnt/ $\beta$ catenin pathway, including Tcf-4, Fra-1, c-Myc, and cyclin D1, were dysregulated in combination therapy. Furthermore, intratumoral administration of LY294002 to subcutaneous LN229 xenograft tumors delayed tumor growth. Our results indicated that the synergistic cytotoxic effect of AD-PTEN and LY294002 is achieved by the inhibition of Wnt/ $\beta$-catenin signaling pathway.

\section{Materials and methods}

Cell culture. Human breast adenocarcinoma MCF-7 cells were provided by Wuhan University Cell Center (Wuhan, China). Cell cultures were incubated at $37^{\circ} \mathrm{C}$ in a $5 \% \mathrm{CO}_{2}$ atmosphere and routinely maintained in Dulbecco's modified Eagle's medium (DMEM) supplemented with $10 \%$ fetal bovine serum (FBS) and $2 \mathrm{mM}$ L-glutamine (Invitrogen, Carlsbad, CA).

Reagents. Recombinant adenoviral vectors containing wildtype PTEN (PTEN sequence, AACTGCTCACCGGAAT) were constructed by the Chinese National Human Genome Center (Beijing, China), at a concentration of $1 \times 10^{10} \mathrm{IU} / \mathrm{ml}$. LY294002 was purchased by Promega. Antibodies against

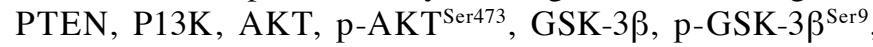
$\beta$-catenin, $\mathrm{p}-\beta$-catenin ${ }^{\text {Ser33 }}$, Fra- 1 , Tcf- 4 , c-myc, PCNA, Bcl-2, cyclin Dl, MMP-2 and MMP-9 were from Santa Cruz Biotechnology, Inc. $\beta$-actin antibody was purchased from Cell Signaling Technology, Inc.

Cell viability assays. Cell viability was determined by MTT (3-(4,5-dimethylthiazol-2-yl)-3,5-diphenyltetrazolium bromide) assays. Briefly, 96-well plates were seeded with $10^{4}$ cells/well $24 \mathrm{~h}$ prior to drug treatment. Cells were treated with LY294002 and/or AD-PTEN at various concentrations for two days. Each experiment was carried out for eight wells. Quantification measurements were obtained at a wavelength of $570 \mathrm{~nm}$ using spectrophotometric analysis. $\mathrm{IC}_{50}$ values were calculated from a linear regression line of the plot of percentage inhibition vs. log inhibitor concentration. The $\mathrm{IC}_{50}$ of LY294002 in MCF-7 cells was used to detect cell viability.

Cell cycle analysis. Cells were grown for $24 \mathrm{~h}$ in normal growth media followed by drug treatment for additional $72 \mathrm{~h}$. After centrifugation, the cell pellets were fixed in $70 \%$ cold ethanol overnight at $4^{\circ} \mathrm{C}$, and then incubated with RNase at $37^{\circ} \mathrm{C}$ for $30 \mathrm{~min}$. The nuclei of cells were stained with propidium iodide for additional $30 \mathrm{~min}$. A total of $10^{4}$ nuclei were examined using a FACSCalibur flow cytometer (BD Biosciences, USA). Samples were analyzed by flow cytometry for the FL-2 area, and DNA histograms were analyzed by Modifit software.

Transwell invasion assays. Cell invasion chambers were prepared by placing $100 \mu \mathrm{l}$ matrigel diluted 1 in 5 onto the filter, and incubating at $37^{\circ} \mathrm{C}$ for $30 \mathrm{~min}$ to allow matrigel polymerization. Cells were removed from culture flasks by trypsinization and resuspended at a concentration of $5 \times 10^{5}$ cells $/ \mathrm{ml}$ in serum-free medium. Of each cell suspension, $2 \mathrm{ml}$ was added to the upper chambers. The chambers were incubated for $48 \mathrm{~h}$, and then fixed and stained with hematoxylin. Five fields of vision were viewed under a light microscope and the cell number under the chamber membrane was counted.

Migration analysis. Briefly, MCF-7 cells (1x10\%/well) were seeded in six-well plates, cultured overnight, and transfected with LY294002 and/or AD-PTEN and the negative control. Upon reaching confluency, the cell layer was scratched with a sterile plastic tip and then immediately washed with growth medium twice and cultured again in DMEM medium (including $10 \% \mathrm{FBS}$ ) for up to $24 \mathrm{~h}$. At different time points, photographic images of the plates were taken under a microscope.

Western blotting. After treatment, cells were lysed in a buffer composed of $50 \mathrm{mM}$ Tris- $\mathrm{HCl}, \mathrm{pH} 7.4,0.1 \mathrm{mM}$ phenylmethylsulfonyl fluoride (PMSF) and $5 \mathrm{mM}$ EGTA for extraction of cellular proteins. The concentration of total proteins was determined by a NanoDrop 2000D (Thermo Scientific, USA). Polyacrylamide gel (SDS-PAGE) electrophoresis was performed on $10 \%$ gels and then transferred onto PVDF membranes. The blots were then immunoblotted with a specific primary IgG antibody overnight at $4^{\circ} \mathrm{C}$, followed by incubation with alkaline horseradish peroxidase-conjugated secondary IgG antibody for 1 hour at room temperature. Blots were developed using enhanced chemiluminescence (ECL) reagents (Amersham Pharmacia, Buckinghamshire, UK) and visualized using the GeneGenius Imaging System (Syngene, Frederick, MD, USA).

In vivo experiments. BALB/C nu 4-week-old female mice were purchased from the Cancer Institute and Hospital, Chinese Academy of Medical Sciences (Beijing, China), and the animal studies were conducted according to institutional ethics guidelines. All mice were maintained under specific pathogen-free conditions. Mice were injected with $10 \mu$ LY294002 once every 4 days. Mice were randomized into five groups ( $n=6$ mice/ group) to receive either vehicle (control, DMSO), LY294002, AD-PTEN or LY294002 and AD-PTEN combined. Mice were sacrificed on Day 28 after tumor cell injections. Tumor growth was assessed weekly by measuring the two greatest perpendicular tumor dimensions. Tumor volume was calculated as follows: tumor volume $\left(\mathrm{mm}^{3}\right)=$ [tumor length $(\mathrm{mm}) \times$ tumor width $\left.(\mathrm{mm})^{2}\right] / 2$.

Immunohistochemistry. Following sacrifice, tumor tissue was removed from the mice and fixed with $4 \%$ formaldehyde for $24 \mathrm{~h}$. Immunostaining was performed on paraffin sections of tumor specimens using the avidin-biotin complex (ABC) method. Briefly, sections were incubated with primary antibody (dilution, 1:200) overnight at $4^{\circ} \mathrm{C}$, then incubated with a biotinylated secondary antibody (dilution, 1:100) at room temperature for $1 \mathrm{~h}$, followed by incubation with $\mathrm{ABC}$-peroxidase reagent and DAB (diaminobenzidine), counterstaining with hematoxylin and visualization under the microscope.

Statistical analysis. Data are expressed as the mean $\pm \mathrm{SE}$. Statistical significance was determined using ANOVA, $\chi^{2}$ test, or Student's t-test using SPSS13.0. Statistical significance was determined at $\mathrm{P}<0.05$. 
A

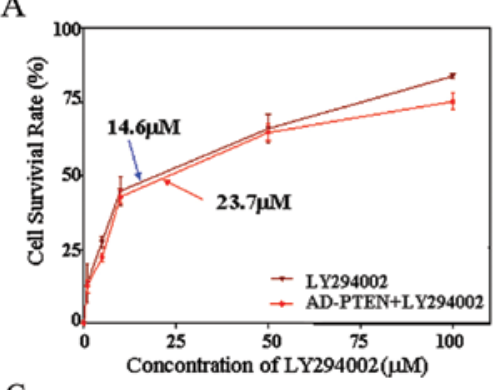

B

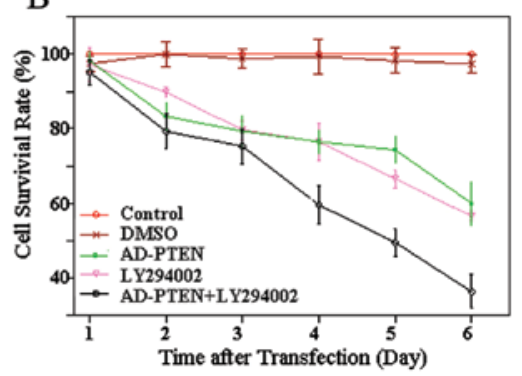

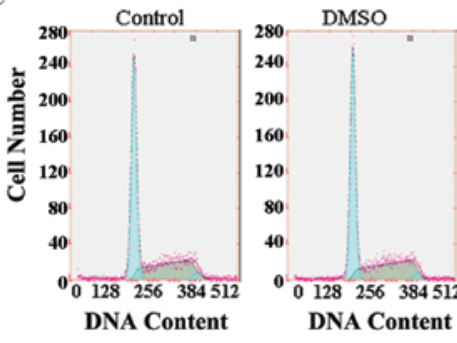
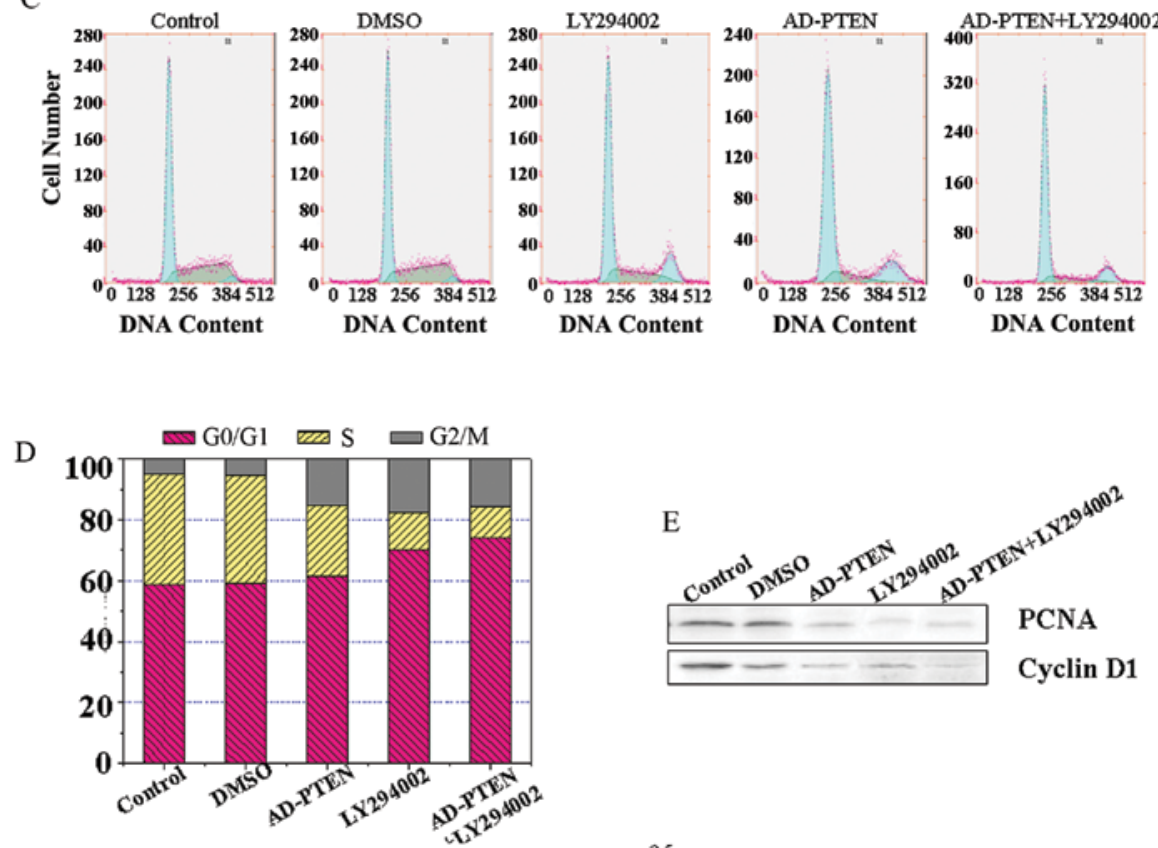

Figure 1. Effect of cell cytotoxicity of LY294002 and AD-PTEN combination treatment in MCF-7 breast cancer cells. (A) IC 50 value of LY294002 was greatly reduced by adding AD-PTEN. (B) Combination therapy suppressed cell growth in vitro. (C) Cell cycle profiles after PI staining. Combination treatment induced $\mathrm{G}_{1}$ arrest in MCF-7 cells. (D) Graphical representation of the cell cycle profiles. (E) PCNA, cyclin D1 expression is significantly decreased by combination therapy.

\section{Results}

AD-PTEN increases LY294002 cytotoxicity in MCF-7 cells. The results indicated that LY294002 can decrease the proliferation of MCF-7 cells, and addition of AD-PTEN can increase the sensitivity of cells to LY294002 treatment. Fig. 1A shows that the LY294002 concentration causing 50\% growth inhibition $\left(\mathrm{IC}_{50}\right)$ of MCF-7 cells is $23.7 \mu \mathrm{mol} / \mathrm{l}$; whereas, in combination with $\mathrm{AD}-\mathrm{PTEN}$ the $\mathrm{IC}_{50}$ was $14.6 \mu \mathrm{mol} / 1$.

To evaluate the synergistic effect of LY294002 and AD-PTEN on cell proliferation, we performed MTT assays in MCF-7 cells transfected with AD-PTEN or treated with LY294002 alone, or both transfected with AD-PTEN and treated with LY294002. Measurements were taken $48 \mathrm{~h}$ after transfection. The data from triplicate samples were analyzed for differences by unpaired, two-tailed t-tests. As indicated, LY294002 alone exhibited a moderate suppressive effect in the first three days of the MTT assay, resulting in maximal inhibition of $60 \%$ in MCF-7 cells (Fig. 1B). The combination of LY294002 and AD-PTEN appeared to show increased suppressive effects in MTT assays. Co-delivery of LY294002 and AD-PTEN consistently maintained the best suppressive effect during the entire MTT assay and resulted in maximal inhibition of $38 \%$ during the six days.
To better understand the synergistic effects on cell cycle progression, we exposed the cells to LY294002 and AD-PTEN alone or in combination, and evaluated changes in cell cycle distribution by flow cytometry analysis (Fig. 1C). Untreated cells served as negative controls. Fig. 1C shows a representative experiment in which $61 \%$ of cells treated with LY29004 were in $\mathrm{G}_{0} / \mathrm{G}_{1}$-phase, whereas treatment with LY294002 and AD-PTEN resulted in $74 \%$ of cells in $\mathrm{G}_{0} / \mathrm{G}_{1}$ phase.

Subsequently, we would like to further explore whether the effects of LY294002 combined with AD-PTEN had any effect on the expression of a set of proteins that are important for cell proliferation and survival. Proliferating cell nuclear antigen (PCNA) is a critical event in growth regulation of breast cancer cells, which acted as a molecular platform to recruit proteins involved in DNA synthesis, cell cycle control, and DNA-damage response and repair. In the current study, a significant decrease in the level of PCNA expression could be observed in the combination treatment group (Fig. 1E). The protein level of PCNA revealed a 5.8-fold reduction in the LY294002-alone-treated cells, and a 7.6-fold reduction in cells treated with LY294002 combined with AD-PTEN-treated cells.

Cyclin D1 is a critical mitogen-regulated cell cycle control element whose transcriptional modulation plays a crucial role in breast cancer growth and progression. The protein level of 


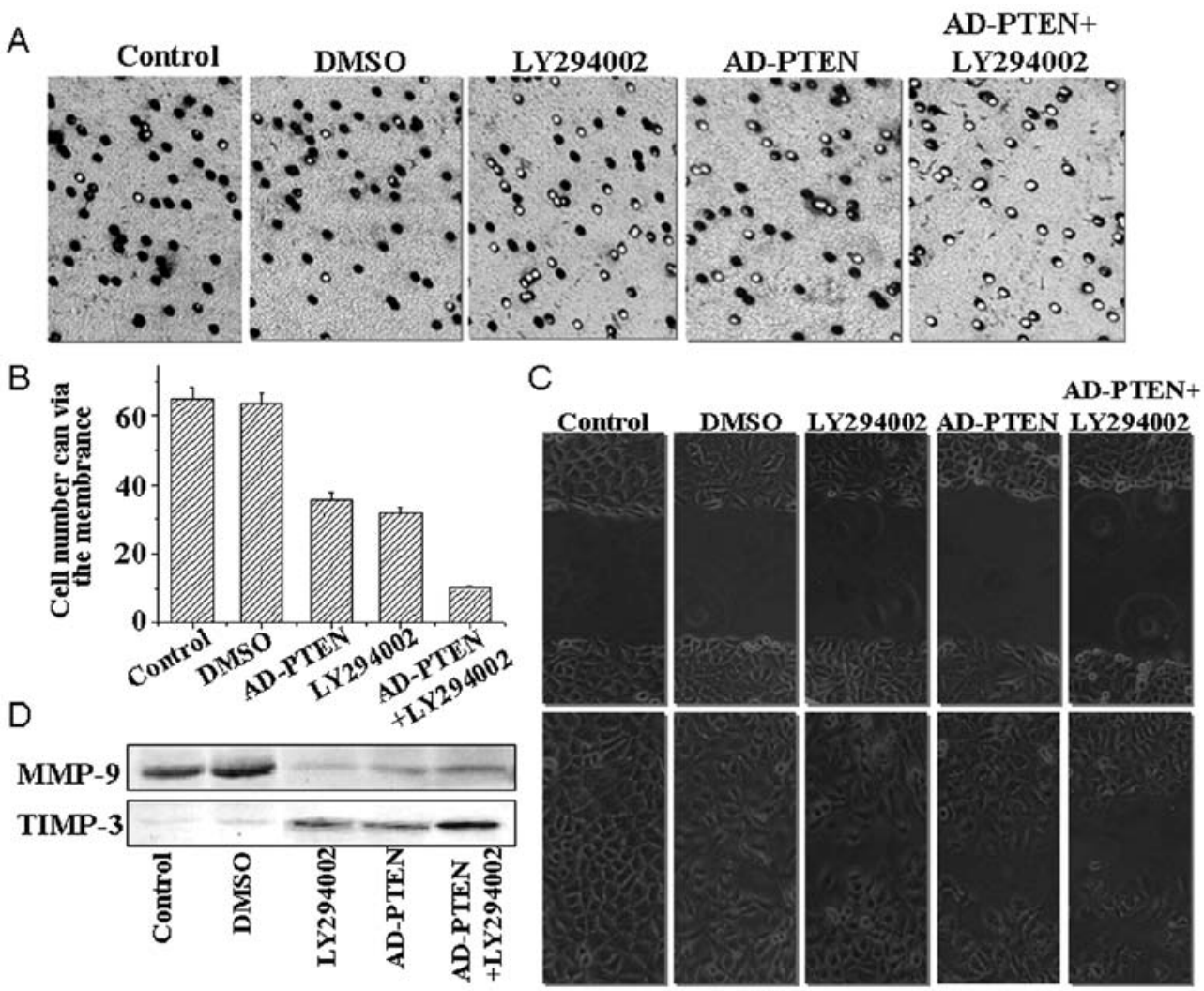

Figure 2. Effects of LY294002 and AD-PTEN on cell invasion and migration ability in the MCF-7 cell line. (A) Cell invasion ability was assessed by transwell assay after a 48-h treatment with LY294002 and AD-PTEN, alone or in combination. (B) The numbers of cells that could invade via the membrane are shown as a histogram. (C) Cell migration ability was determined by wound-healing assay. (D) Western blotting validation of proteins (MMP-2 and TIMP-3) expression in combination therapy in the MCF-7 cell line.

cyclin D1 revealed a 4.2-fold reduction in single LY294002treated cells and a 5.8-fold decrease in the combined treatment group, respectively (Fig. 1E). These findings indicate that, at least in vitro, AD-PTEN sensitizes breast cancer cells to LY294002 cytotoxicity.

LY294002 and AD-PTEN combination therapy regulates cell invasion and migration. To measure the effects of LY294002 combined with AD-PTEN on breast cancer cell invasiveness and migration, we employed the transwell invasion assay and a xenograft model, which are better indicators of cancer migratory and invasive properties in vitro. Transwell assays consist of two fluid-filled, stacked compartments separated by a porous membrane filter coated with matrigel. Cells were grown in the upper chamber and assessed for invasion through the matrigel toward a chemo-attractant (10\% serum) in the lower chamber. The number of invasive cells in cultures treated with LY294002 and AD-PTEN was reduced relative to the control cells without any treatment (Fig. 2A); a decrease of 65 to 31-35 MCF-7 cells. The number of invasive cells in cultures with combination treatment was significantly reduced relative to the cells treated with LY294002 or AD-PTEN alone (Fig. 2B); a decrease from 31-35 to 10 MCF-7 cells.

The number of migrating cells in LY294002 and AD-PTEN combination treatment group were 43 (Fig. 2C), which was significantly fewer than the LY294002 treated group $(n=134)$, the AD-PTEN treated group $(\mathrm{n}=102)$ and the control group $(n=352)$.
Proteins of the matrix metalloproteinase (MMP) family are involved in the breakdown of extracellular matrix, which contributes to tumor cell invasion of normal tissues and metastasis. In this respect, the levels of expression of TIMP-3 and MMP-9 proteins after combination treatments were evaluated by western blotting (Fig. 2D). Significantly decreased expression of was observed after treatment with LY294002 combined with AD-PTEN, while the level of TIMP-3 protein was dramatically enhanced in the combination treatment group compared to the single LY294002-treated group.

Combination treatment with LY294002 and AD-PTEN significantly decreases $A K T^{\text {Ser } 473}$ and $G S K-3 \beta^{\text {Ser9 } 9}$ phosphorylation in breast cancer cells. To understand the molecular mechanisms by which LY294002 combined with AD-PTEN resulted in decreased proliferation, invasion and migration in MCF-7 breast cancer cells, the levels of phosphorylated $\mathrm{AKT}^{\mathrm{Ser} 473}$ and GSK-3 $\beta^{\text {Serg }}$ were compared after 12-h single or combined treatment with LY294002 or/and AD-PTEN. As shown in Fig. 3, both phosphorylated AKT ${ }^{\text {Ser473 }}$ and GSK-3 $\beta^{\text {Ser9 }}$ were decreased by single LY294002 treatment or single AD-PTEN treatment. After combination treatment with LY294002 and AD-PTEN, both phosphorylated AKT ${ }^{\text {Ser473 }}$ and GSK-3 $\beta^{\text {Ser9 }}$ were significantly decreased.

To detect whether decreased proliferation, invasion and migration in MCF-7 breast cancer cells caused by combined treatment of LY294002 and AD-PTEN was modulated by GSK-3 $/ \beta$-catenin signaling pathway, cell cycle regulators 


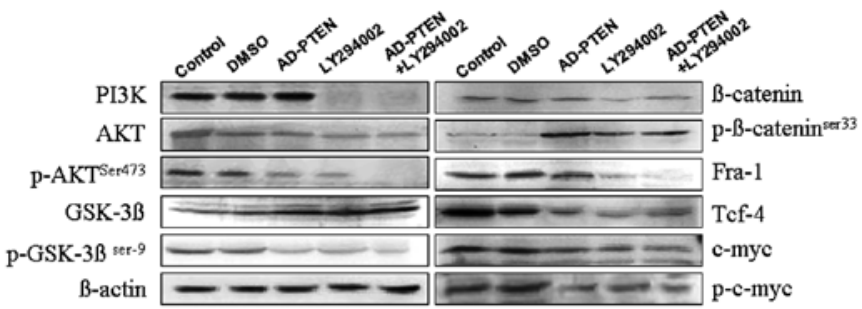

Figure 3. Combination treatment with LY294002 and AD-PTEN remarkably decreased AKT and GSK- $3 \beta$ phosphorylation in MCF-7 breast cancer cells. MCF-7 cells were treated with PI3K inhibitor LY294002, or/and AD-PTEN for $48 \mathrm{~h}$. Western blotting with antibodies against AKT(pan), phosphorylated $\mathrm{AKT}^{\mathrm{Ser} 473}$, GSK-3 $\beta$, phosphorylated GSK-3 $\beta^{\text {Ser9 }}, \beta$-cantenin, p- $\beta$-catenin ${ }^{\text {Ser33 }}$, and followed by densitometry quantification. Fra-1, Tcf-4, c-myc expression were all decreased in the combination treatment group.

such as c-Myc and cyclin D1, and transcription factors such as Fra-1 and Tcf-4, were measured by western blotting. As shown in Fig. 3, combined treatment could significantly inhibit downstream $\beta$-catenin signal transduction pathways, compared with LY294002 or AD-PTEN single treatments.

LY294002 combined with AD-PTEN suppresses breast cancer growth in the xenograft model. LY294002 combined with AD-PTEN demonstrated an excellent tumor suppressive effect and may be a potential therapy for human breast cancer. To further confirm this hypothesis, four experimental groups were examined in an MCF-7 xenograft model: i) control, ii) LY294002, iii) AD-PTEN and iv) combined AD-PTEN and LY294002 therapy groups. At the beginning of treatment, the mean tumor volumes of the mice in LY294002 and AD-PTEN treated groups alone or in combination were 120, 127 and $136 \mathrm{~mm}^{3}$, respectively, with no statistically significant differences among these three groups. The mice were monitored every 4 days for 4 weeks, and the tumor volume of mice in each group was measured and compared. At the end of the experiment, significant decreases in tumor volume was only observed in the combination treatment group; the tumor volume was greatly reduced compared with the LY294002 group ( $763 \mathrm{~mm}^{3}$ vs. $1789 \mathrm{~mm}^{3}$ and $\left.1433 \mathrm{~mm}^{3}, \mathrm{P}<0.01\right)$. Consistent with the in vitro results, we observed decreased expression of phosphorylated AKT as well as PCNA, cyclin D1 and MMP-9 in the combination-treated tumors (Fig. 4B).

\section{Discussion}

Selection of appropriate combinations of anticancer agents that can exert synergistic cytotoxic interactions has been widely adopted and utilized in preclinical and clinical studies. Yang et al (12) revealed that temsirolimus treatment combined with either BEZ235 (a dual PI3K/mTOR inhibitor) or ZSTK474 (a pan PI3K inhibitor) inhibited phosphorylation of both 4E-BP1 and the substrate for S6K, ribosomal S6 (rS6), which ultimately resulted in synergistic cell death of six endometrial cancer cell lines. Morelli et al (13) demonstrated that dual inhibition of MAPK signaling and HDAC activity resulted in inhibition of several colorectal cancer biological processes, which was mediated by an increased level of caspase 3/7 activity, cleaved PARP and perhaps increased acetylated histone H3 levels. Recent studies in human gastric
A

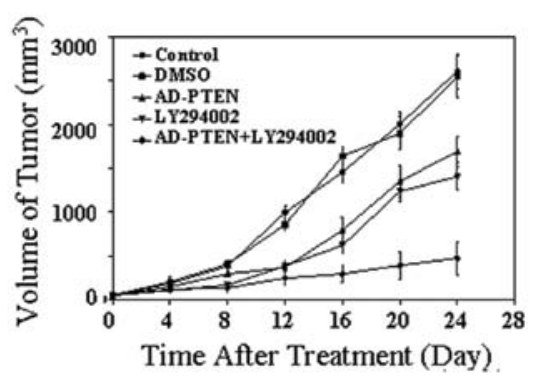

B

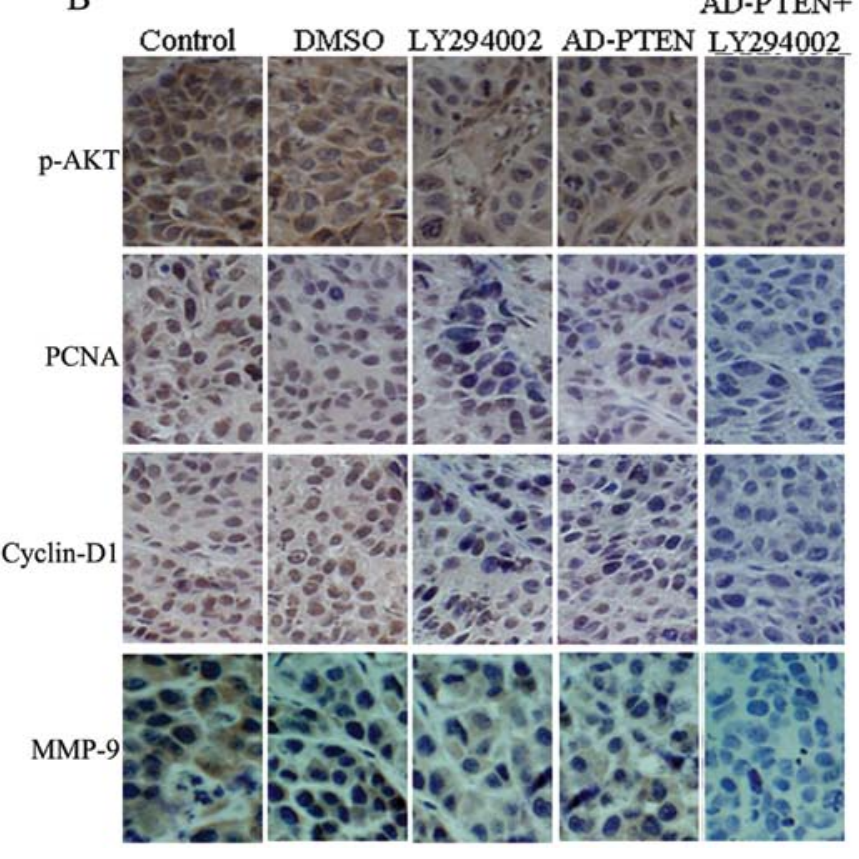

Figure 4. MCF-7 breast cancer cell xenograft tumor experiment. (A) Growth curves of MCF-7 cell xenografts with LY294002, AD-PTEN alone or in combination. (B) Immunohistochemistry analysis of MCF-7 xenograft tumors. p-AKT, PCNA, cyclin D1 and MMP-9 expression was dramatically suppressed in combination treatment xenograft tumors.

cancer indicated that DLL4 inhibitor combined with Jagged1siRNA gene therapy mediated by adenovirus can enhance inhibition of SGC7901 human gastric cancer cell proliferation and invasion through the DLL4/ Jagged1-Notch1 pathway (14).

Despite the availability of several active combination regimens for breast cancer, the 5-year survival rate remains poor, supporting the development of novel therapeutic approaches. The PI3K/AKT signaling pathway is almost universally dysregulated in breast cancer, with specific occurrence of PTEN mutations, which results in overactivation of PI3K/AKT signaling and its downstream signal transduction pathways $(15,16)$. Therefore, it has become an attractive target for cancer treatment and as a result, inhibition of this pathway is a major strategy for cancer therapy.

However, single-targeted therapeutics against the PI3K/AKT pathway have demonstrated only modest clinical benefits. In the present study, we compared the pro-apoptotic effect of treatment with LY294002, a specific PI3K inhibitor, and AD-PTEN, a recombinant adenovirus-PTEN, alone or in combination. Our results revealed that $\mathrm{AD}-\mathrm{PTEN}$ significantly reduced the $\mathrm{IC}_{50}$ of LY294002 concentration by 2-fold compared to LY294002 alone. Cell proliferation, migration, and invasion ability were 
also dramatically inhibited by combination treatment compare to individual LY294002 or AD-PTEN treatment.

To understand the underlying molecular mechanisms, we then investigated the activation of downstream effectors of the PI3K signaling pathway. Expression of total AKT was not

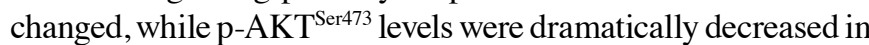
combination therapy compared to LY294002 alone. Importantly, combined treatment resulted in lower levels of phosphorylation GSK-3 $\beta^{\text {Ser9 }}, \beta$-catenin, c-Myc and Tcf-4 (Fig. 3). GSK-3 $\beta$ is the main target for AKT and is inhibited by AKT phosphorylation. In the Wnt $/ \beta$-catenin signaling pathway, GSK-3 $\beta$ directly determines the stabilization of $\beta$-catenin by phosphorylation (17). As a result, there is an accumulation of stabilized hypophosphorylated $\beta$-catenin, which then translocates to the nucleus and associates with transcription factors of the Lef/Tcf family to initiate the expression of a broad range of genes, such as c-Myc and cyclin D1. Thus, our data indicated that the Wnt $/ \beta$-catenin signaling pathway may be responsible for the synergistic cytotoxic effect mechanism of LY294002 and AD-PTEN.

We showed for the first time that inactivation of PI3K/AKT represses $\beta$-catenin-mediated transcription in breast cancer. Indeed, this hypothesis of cross-talk between the $\beta$-catenin and PI3K/AKT signaling pathways might have supportive evidence. Damsky et al (18) demonstrated that $\beta$-catenin is a central mediator of melanoma metastasis to the lymph nodes and lungs, controls tumor differentiation and regulates both MAPK/ERK and PI3K/AKT signaling. In U87MG cells, Zhang et al (19) also showed that the activation of Notch1 by DLL4 stimulation or by the overexpression of Notch intracellular domain (NICD) resulted in AKT activation, thereby promoting $\beta$-catenin activity. It has also been reported that the cooperation between $\mathrm{Wnt} / \beta$ catenin and PTEN/PI3K/AKT signaling promotes primitive hematopoietic stem cell self-renewal and expansion (20).

Taken together, our data demonstrate that AD-PTEN significantly enhances the sensitization of breast cancer cells to LY294002. We also showed that increased GSK-3 $\beta$ activity resulting in the inhibition of $\beta$-catenin signaling pathway may be responsible for the synergistic cytotoxic effect of LY294002 and AD-PTEN. Thus, inhibition of PI3K was proven to be an effective strategy for inactivation of the Wnt/ $\beta$-catenin pathway. Furthermore, we also showed for the first time that cross-talk between PI3K/AKT and Wnt/ $\beta$-catenin pathway occurs in breast cancer. The findings of the present study might help guide the development of potential therapeutic targets for the treatment of breast cancer patients.

\section{Acknowledgements}

This study was financially supported by the 973 Programme Grant (2009CB918903, 2011CB933100) from the Ministry of Science and Technology of China, the China National Natural Scientific Fund (51073118, 81101916, 51103107), the Tianjin Science and Technology Committee (10JCYBJC12500,10JCZDJC19700), and the Program for New Century Excellent Talents in University (NCET-08-0393).

\section{References}

1. Ferté C, André F and Soria JC: Molecular circuits of solid tumors: prognostic and predictive tools for bedside use. Nat Rev Clin Oncol 7: 367-380, 2010.
2. Sun $\mathrm{CH}$, Chang $\mathrm{YH}$ and Pan CC: Activation of the PI3K/Akt/ mTOR pathway correlates with tumour progression and reduced survival in patients with urothelial carcinoma of the urinary bladder. Histopathology 58: 1054-1063, 2011.

3. Mazzoletti M, Bortolin F, Brunelli L, Pastorelli R, Di Giandomenico S, Erba E, Ubezio P and Broggini M: Combination of PI3K/mTOR inhibitors: antitumor activity and molecular correlates. Cancer Res 71: 4573-4584, 2011.

4. Chen Y, Wang BC and Xiao Y: PI3K: A potential therapeutic target for cancer. J Cell Physiol 227: 2818-2821, 2011.

5. Ghayad SE and Cohen PA: Inhibitors of the PI3K/Akt/mTOR pathway: new hope for breast cancer patients. Recent Pat Anticancer Drug Discov 5: 29-57, 2010.

6. Liu W, Zhou Y, Reske SN and Shen C: PTEN mutation: many birds with one stone in tumorigenesis. Anticancer Res 28: 3613-3619, 2008.

7. Heikkinen T, Greco D, Pelttari LM, Tommiska J, Vahteristo P, Heikkila P, Blomqvist C, Aittomaki $\mathrm{K}$ and Nevanlinna $\mathrm{H}$ : Variants on the promoter region of PTEN affect breast cancer progression and patient survival. Breast Cancer Res 13: R130, 2011.

8. Carraway $\mathrm{H}$ and Hidalgo M: New targets for therapy in breast cancer: mammalian target of rapamycin (mTOR) antagonists. Breast Cancer Res 6: 219-224, 2004

9. Lai JP, Sandhu DS, Yu C, Moser CD, Hu C, Shire AM, Aderca I, Murphy LM, Adjei AA, Sanderson S and Roberts LR: Sulfatase 2 protects hepatocellular carcinoma cells against apoptosis induced by the PI3K inhibitor LY294002 and ERK and JNK kinase inhibitors. Liver Int 30: 1522-1528, 2010.

10. Shin JY, Kim JO, Lee SK, Chae HS and Kang JH: LY294002 may overcome 5-FU resistance via down-regulation of activated p-AKT in Epstein-Barr virus-positive gastric cancer cells. BMC Cancer 13: 425, 2010.

11. Fujiwara M, Izuishi K, Sano T, Hossain MA, Kimura S, Masaki T and Suzuki Y: Modulating effect of the PI3-kinase inhibitor LY294002 on cisplatin in human pancreatic cancer cells. J Exp Clin Cancer Res 27: 76-84, 2008.

12. Yang S, Xiao X, Meng X and Leslie KK: A mechanism for synergy with combined mTOR and PI3 kinase inhibitors. PLoS One 6: e26343, 2011

13. Morelli MP, Tentler JJ, Kulikowski GN, Tan AC, BradshawPierce EL, Pitts TM, Brown AM, Nallapareddy S, Arcaroli JJ, Serkova NJ, et al: Preclinical activity of the rational combination of Selumetinib (AZD6244) in combination with Vorinostat in KRAS mutant colorectal cancer models. Clin Cancer Res 18: 1051-1062, 2012.

14. Sun HW, Wu C, Tan HY and Wang QS: Combination DLL4 with Jagged1-siRNA can enhance inhibition of the proliferation and invasiveness activity of human gastric carcinoma by Notch1/ VEGF pathway. Hepatogastroenterology 59: 115-116, 2011.

15. Adamo B, Deal AM, Burrows E, Geradts J, Hamilton E, Blackwell KL, Livasy C, Fritchie K, Prat A, Harrell JC, et al: Phosphatidylinositol 3-kinase (PI3K) pathway activation in breast cancer brain metastases. Breast Cancer Res 13: R125, 2011.

16. Miller TW, Rexer BN, Garrett JT and Arteaga CL: Mutations in the phosphatidylinositol 3-kinase pathway: role in tumor progression and therapeutic implications in breast cancer. Breast Cancer Res 13: 224, 2011.

17. Dozza B, Smith MA, Perry G, Tabaton M and Strocchi P: Regulation of glycogen synthase kinase-3beta by products of lipid peroxidation in human neuroblastoma cells. J Neurochem 89: 1224-1232, 2004.

18. Damsky WE, Curley DP, Santhanakrishnan M, Rosenbaum LE, Platt JT, Gould Rothberg BE, Taketo MM, Dankort D, Rimm DL, McMahon $\mathbf{M}$ and Bosenberg $\mathrm{M}$ : $\beta$-catenin signaling controls metastasis in Braf-activated Pten-deficient melanomas. Cancer Cell 20: 741-754, 2011.

19. Zhang X, Chen T, Zhang J, Mao Q, Li S, Xiong W, Qiu Y, Xie Q and Ge J: Notch1 promotes glioma cell migration and invasion by stimulating $\beta$-catenin and NF- $\kappa \mathrm{B}$ signaling via AKT activation. Cancer Sci 103: 181-190, 2011.

20. Perry JM, He XC, Sugimura R, Grindley JC, Haug JS, Ding S and Li L: Cooperation between both Wnt/ $\beta$-catenin and PTEN/ $\mathrm{PI} 3 \mathrm{~K} / \mathrm{Akt}$ signaling promotes primitive hematopoietic stem cell self-renewal and expansion. Genes Dev 25: 1928-1942, 2011. 